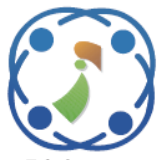

\title{
Dysgraphia Classification based on the Non-Discrimination Regularization in Rotational Region Convolutional Neural Network
}

\author{
Fathima Ghouse $^{1 *}$ \\ Kavitha Paranjothi ${ }^{2}$ \\ Revathi Vaithiyanathan ${ }^{3}$ \\ ${ }^{I}$ Department of Computer Science and Engineering, Adhiyamaan College of Engineering, Hosur, Tamilnadu, India \\ ${ }^{2}$ Department of Computer Science and Engineering, CMR Institute of Technology, Bengaluru, India \\ ${ }^{3}$ Department of Computer Science and Engineering, Dayananda Sagar University, Bengaluru, India \\ * Corresponding author's Email: fathima.ace@gmail.com
}

\begin{abstract}
Dysgraphia is a handwriting disorder and the classification of dysgraphia in children's handwritten images helps to identify the dysgraphia patient effectively and also prevents low self-esteem. Traditional methods of dysgraphia classification based on experts manually classify the dysgraphia that requires more cost and time. Few researches apply the machine learning and deep learning techniques for the classification of dysgraphia and existing models have the limitations of overfitting problems in the training process. In this research, the Non-Discrimination Regularization in Rotational Region Convolutional Neural Network (NDR-R2CNN) is proposed to improve the efficiency of dysgraphia classification. The balancing parameters are introduced in the loss function to balance the class in the training and eliminate the features to reduce the overfitting problem. The collected children's handwriting data were used to evaluate the performance of the proposed NDR-R2CNN model. The proposed NDR-R2CNN model has the advantages of effective feature analysis and non-discrimination word analysis. The NDR-R2CNN model has the accuracy of $98.2 \%$ and the SMOTE+SVM method has $90.2 \%$ accuracy in Dysgraphia classification. The result shows that the NDR-R2CNN model has $98.2 \%$ accuracy and the existing CNN has the accuracy of $94.2 \%$.
\end{abstract}

Keywords: Dysgraphia, Handwriting data, Non-discrimination regularization, Overfitting and rotational region convolutional neural network.

\section{Introduction}

Handwriting is still used in academics, communication, and self-expression, despite technological development. Writing is a major occupation in childhood and this required participation in a variety of environments, including home, classroom and society at large [1]. Handwriting is a complex task that involves perceptual components, motor, linguistic and cognitive. Dysgraphia is finding difficulty in handwriting performance and studies show that this is quite frequent between 12 to $33 \%$ in school-age children that involves in illegible writing and dysfluent writing comprising communication skills, self-expression and the possibility to record their ideas [2]. These difficulties not only affect the child's school performance and also affect the child's self- esteem and everyday life in the future [3]. Children's timely development of writing and reading skills is important in behavioral and academic progress. Dysgraphia is also considered as a handwriting learning disability that is related to attention deficit disorder, developmental coordination disorder, and dyslexia, which are all neurodevelopmental disorders. The everyday life of the children is seriously impaired by dysgraphia and children require therapeutic care. Early classification of dysgraphia is of great importance in pediatrics and this helps to take necessary care for the children $[4,5]$.

Some of the symptoms of dysgraphia are involved in the written letter is not ideal, incorrect holding of ballpoint pen or pencil, the letters go up and down, slow writing and the writing seems thin [6]. A recent study highlights six specific handwriting features for identification of dysgraphia in children and handwriting disorder [7]. Machine learning is a 
common approach for image and signal classification due to its efficiency. Machine learning is applied for the classification of handwriting text line images and speech signals [8]. The existing methods of TestGraphia and CNN have lower efficiency in feature analysis and non-discrimination is not analyzed in the existing models $[9,10]$. In this research, the NDR-R2CNN model is proposed to improve the efficiency of the dysgraphia classification. The collected children's data were used to evaluate the performance of the proposed model. The existing R2CNN model considers the incomplete data into one class and this affects the performance. The NDR is applied to complete the input that helps to compare with a target for a better understanding of features. The balancing parameters in NDR are applied in the loss function to balance the class in the training and eliminate the features to reduce overfitting. The proposed method provides effective feature analysis and non-discriminative word analysis to improve efficiency.

This paper is organized as follows: a review of recent methods in dysgraphia classification is given in section 2 and the proposed method is presented in section 3. The simulation setup is explained in section 4 and the result of the proposed model is given in section 5. The conclusion of this research is given in section 6 .

\section{Literature review}

Dysgraphia is a handwriting disorder and early detection of dysgraphia helps to treat the patient effectively. Few researches involve in the classification of dysgraphia using the data-driven method and machine learning techniques.

Dimauro [11] developed the software of TestGraphia to support the doctor for diagnosis of dysgraphia among the patient. Various document analysis algorithms and feature selection algorithms were developed to detect dysgraphia. Children handwriting of $2^{\text {nd }}$ grade to $5^{\text {th }}$ grade were collected to evaluate the TestGraphia software. The 9 algorithms were used to analyze the features of the text from the input data. The result shows that developed software has higher performance in detecting dysgraphia and the computational time of the model is less. The developed model has ambiguity in borderline removal due to the presence of variance and non-discriminatory letters were not analyzed in the software.

Gazda [12] applied convolutional neural network $(\mathrm{CNN})$ for the diagnosis of parkinson's disease from handwriting images. The pretrained CNN model was applied for the idea of multiple fine turning to bridging the gap between the source and target dataset. A more efficient transfer learning method was applied in the CNN model to improve the performance of the detection. The result shows that the developed model has a higher performance in the detection compared to an existing model. The model creates the overfitting problem in training due to the low dropout rate and fine-tuning of the model is required to overcome this limitation.

Mucha [13] applied the fractional-order deviation method for the detection of dysgraphia among parkinson's patients. Kinematic partial correlation analysis of pearson's and spearman's measures was used to analyze the relationship between the designed features and the patient's clinical data. The 33 parkinson's disease persons and 36 healthy people handwriting were collected to evaluate the performance of the proposed model. A regression model was applied to train the features and detect dysgraphia among the patients. The result shows that the proposed model has higher performance compared to the existing methods. The kinematic measures are not sufficient for analyzing the discrimination of fractional order deviation features.

Asselborn [14] analyzed a scale of handwriting difficulties from the lightest case to severe case, and compare the score of the same age and gender. The principal component analysis (PCA) method was applied to reduce the set of 53 handwriting features to three dimensions. The clustering method was applied in the data set with three axes to accurate detection of dysgraphia detection. The shape features were effectively analyzed in the method and the clustering method effectively classifies the data. The result shows that the developed model has a higher performance in dysgraphia detection. The clustering method has an overfitting problem due to the training of many features.

Lamba [15] proposed a kinematic feature extraction method from the handwritten document for the detection of parkinson's disease. The synthetic minority oversampling technique (SMOTE) method was applied to handle the imbalanced dataset. A total of 29 kinematics features was extracted from the dataset for detection. The genetic algorithm and mutual information gain were applied for the feature selection process. The classifiers such as XGBoost, AdaBoost, random forest, and support vector machine (SVM) were applied for the detection of parkinson's disease. The 10-fold cross-validation was applied to evaluate the performance of the model. The SMOTE method doesn't consider the neighborhood value and this involves the overlapping of features. The SVM has lower performance in overlapping and imbalanced datasets. 


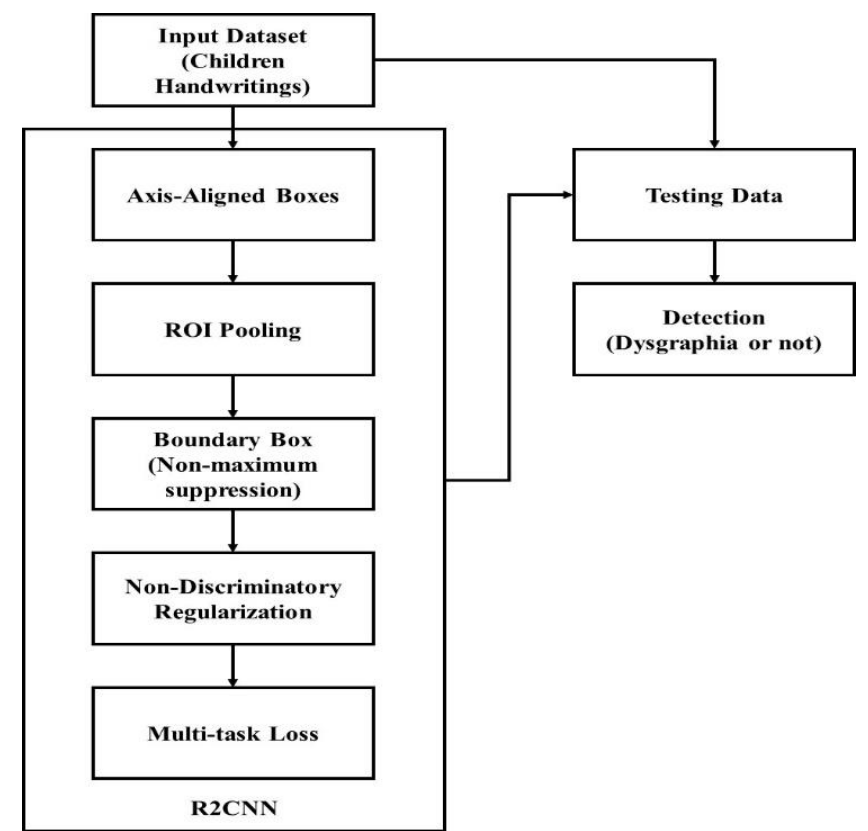

Figure. 1 The overview of the proposed NDR-R2CNN model

\section{Methodology}

Early detection of dysgraphia helps to treat the patient effectively and also prevents the patient from low self-esteem. Few existing methods such as TestGraphia and CNN were applied for the classification of dysgraphia and have the limitation of overfitting problem.

In this research, the NDR-R2CNN model has been proposed to improve the performance of dysgraphia classification. The children's handwriting collected datasets were used to evaluate the performance of the developed method. The overview of the developed NDR-R2CNN model is shown in Fig. 1.

\subsection{Rotational region CNN (R2CNN)}

Region proposal and region classification are popular two-stage objection classification methods adopted in convolutional neural network $(\mathrm{CNN})$. faster R-CNN is used to develop the rotational region convolutional neural network (R2CNN) $[16,17]$. The region proposal network (RPN) is applied to generate text region proposals for axis-aligned bounding boxes to enclose the arbitrary-oriented texts. Several ROI poolings with various pooled sizes $(7 \times 7$, $11 \times 3,3 \times 11$ ) are applied for each proposal on convolution feature maps and pooled features are applied for classification. Inclined minimum area boxes, axis-aligned boxed and text/non-text scores are predicted based on the fully connected layers and pooled features. As post-processed, inclined nonmaximum suppression is processed on the inclined boxes to get detection results. The pooling layer size is calculated based on the average pooling and the feature map.

\subsection{Axis-aligned boxes}

Axis-aligned bounding boxes are generated by the RPN to enclose the arbitrary-oriented texts. The text in the axis-aligned box belongs to one of the following: (a) horizontal direction text, (b) vertical direction text, (c) diagonal direction text. Arbitraryoriented texts enclose axis-aligned boxes are generated based on the RPN.

There are small texts in the images compared to general objects and RPN small anchor scale is applied to support the small texts. The Faster R-CNN original anchor scales are $(8,16,32)$ involves in two methods: (a) anchor scales size is changed into smaller $(4,8$, 16); (b) a new anchor scale is added $(4,8,16,32)$. The analysis shows that a smaller anchor is helpful for small text detection in the model.

RPN's other parameter settings are similar to faster R-CNN [18], including positive samples and negative samples definition, anchor aspect ratio, etc.

\subsection{ROI poolings of different pooled sizes}

For each RPN proposal, the faster R-CNN method performs ROI pooling on the feature map in the pooled size of $7 \times 7$. Three ROI poolings with various sizes are applied to some texts with higher widths than their heights to observe more text characteristics. The pooled features are applied for further processes i.e., two pooled sizes such as $11 \times 3$ and $3 \times 11$ are added specifically. The more 
horizontal features are analyzed on pooled size of $3 \times$ 11 and help horizontal text detection with width higher than its height. The more vertical features are analyzed with a pooled size of $11 \times 3$ and this is applied for vertical text detection with higher height than its width.

\subsection{Boundary box}

After RPN involves axis-aligned bounding boxes to refine inclined bounding boxes and arbitraryoriented texts, RPN generated a proposal region to classify as text or non-text. Axis-aligned box is related to each inclined box and inclined bounding boxes are targets. Adding an axis-aligned bounding box improves the performance.

\subsection{Non-maximum suppression}

In the post-process detection, the candidates are performed based on non-maximum suppression (NMS) for current object detection methods. Normal NMS or axis-aligned bounding boxes or inclined bounding boxes are estimated in this step. The traditional classification of intersection-over-union (IoU) is modified in inclined NMS to be IoU between two inclined bounding boxes. The IoU calculation method is used [19].

\subsection{Non-discriminatory regularization}

Applying the NDR model in R2CNN helps to complete the information based on the probability of previous data. Once the suppression detects the handwritten letters and NDR method is applied to complete the information. The existing R2CNN model considers the incomplete data as another class and this affects the performance. The NDR method is applied to complete the input data and apply for classification. All off-diagonal elements are pushed to zero to obtain $G_{l}^{c} \rightarrow I \forall c$ in the following regularization method, as shown in Eq. (1). The $I$ is the identity matrix, and $G_{l}^{c}$ is a full rank of $l^{\text {th }}$ layer and $c^{\text {th }}$ class.

$$
R_{l}^{\text {strong }}=\frac{1}{C} \sum_{c} \frac{1}{2 M^{c}} \sum_{i \neq j}\left|g_{l, i, j}^{c}\right|
$$

Where data cardinality $M^{c}$ is belongs to the same discrimination class $c$. The input and dictionary data patterns are $i$ and $j$, respectively. A hard constraint is having $R_{l}^{\text {strong }} \rightarrow 0$. Effective conditions based on the average correlations impose a weaker, as shown in Eq. (2).

$$
R_{l}=\frac{1}{C} \sum_{c} \frac{1}{2 M^{c}}\left|\sum_{i \neq j} g_{l, i, j}^{c}\right|
$$

The weaker and strong conditions of ReLUactivated networks coincide as $g_{l, i, j}^{c} \geq 0 \forall_{i, j}$. This regularization provides the completion of words that helps to compare with target data to analyze the difference.

\subsection{Training objectives (multi-task loss)}

The RPN training loss is similar to faster R-CNN [20]. The R2CNN loss function is introduced on each RPN-generated proposal of an axis-aligned box.

Each region loss function is based on the summation of the text/non-text box regression loss and classification loss. The box regression loss consists of two parts: axis-aligned boxes loss that encloses the arbitrary-oriented texts and minimum area boxes inclined loss. Each proposal multi-task loss function is denoted as in Eq. (3).

$$
\begin{aligned}
& L\left(p, t, v, v^{*}, u, u^{*}\right)=L_{c l s}(p, t)+ \\
& \lambda_{1} t \sum_{i \in\{x, y, w, h\}} L_{r e g}\left(v_{i}, v_{i}^{*}\right)+ \\
& \lambda_{2} t \sum_{i \in\left\{x_{1}, y_{1}, x_{2}, y_{2}, h\right\}} L_{r e g}\left(u_{i}, u_{i}^{*}\right)
\end{aligned}
$$

Three terms trade-off is control based on the balancing parameters of $\lambda_{1}$ and $\lambda_{2}$. These balance parameters help to maintain the class in the dataset and also reduces the overfitting problem.

The box regression is performed on the text and the class label indicator is denoted as $t$. The background is labelled as $0(t=0)$ and the text is labeled as $1(t=1)$. Softmax function computed the probability of text and background classes with parameter $p=\left(p_{0}, p_{1}\right)$. True class $t \log \operatorname{loss}$ is denoted as $L_{c l s}(p, t)=-\log p_{t}$.

Bounding box regression targets of the true axisaligned tuple are denoted as $v=\left(v_{x}, v_{y}, v_{w}, v_{h}\right)$, including center point coordinates, height, width and text label predicted tuple $v^{*}=v x^{*}, v y^{*}, v w^{*}, v h^{*}$. Bounding box regression targets of a truly inclined tuple are $u=\left(u_{x 1}, u_{y 1}, u_{x 2}, u_{y 2}, u_{h}\right)$ including first two points coordinates of its heights and the inclined box and text label predicted tuple is denoted as $u^{*}=u_{x 1}^{*}, u_{y 1}^{*}, u_{x 2}^{*}, u_{y 2}^{*}, u_{h}^{*}$. A scale-invariant translation is denoted as $v$ and $v^{*}$ for parameterization and object proposal of height-width in shift, relative log-space is used. The parameterization of $u_{x 1}, u_{y 1},\left(u_{x 2}, u_{y 2}\right)$, $\left(u_{x 1}^{*}, u_{y 1}^{*}\right)$ is used for inclined bounding boxes and the same width of $u_{x 2}^{*}, u_{y 2}^{*}$ is $v_{x}, v_{y}$. The parameterization of $u_{h}$ and $u_{h}^{*}$ are similar to the parameterization of $v_{h}$ and $v_{h}^{*}$.

Let $w, w^{*}$ denotes $L_{r e g} w, w^{*}, v_{i}, v_{i}^{*}$ or $u_{i}, u_{i}^{*}$, as given in Eq. $(4,5)$. 


$$
\begin{aligned}
& L_{\text {reg }}\left(w, w^{*}\right)=\operatorname{smooth}_{L 1}\left(w-w^{*}\right) \\
& \text { smooth }_{L 1}(x)=\left\{\begin{array}{cc}
0.5 x^{2} & \text { if }|x|<1 \\
|x|-0.5 & \text { otherwise }
\end{array}\right.
\end{aligned}
$$

The classification is performed on the input dataset based on this training and testing process.

\section{Simulation setup}

This section describes the dataset, system configuration, parameter settings and metrics.

Dataset: The dataset was collected from primary schools in top cities in tamil nadu and karnataka with help of occasional therapists. The 150 samples are taken to build the model from the children age 6 to 8 from primary school. The sample images of normal and dysgraphia samples from collected and standard datasets were shown in Fig. 2 (a - c). In Fig. 2 (b), 'The' in the sentence is not written properly and the shape of the word 'was' is not proper. In Fig. 2 (c), the shape of the letters is not properly written. The standard dygraphia dataset [9] was used to evaluate the performance of the proposed and existing model. The standard dysgraphia dataset is available in https://github.com/peet292929/Dysgraphiadetection-through-machine-learning.

Metrics: The four-parameter metrics such as accuracy, precision, recall and f-measure were evaluated from the proposed model. The formulas for accuracy, precision, recall and f-measure are shown in Eq. (6 - 9), respectively.

$$
\begin{aligned}
& \text { Accuracy }=\frac{T P+T N}{T P+T N+F P+F N} \times 100 \\
& \text { Precision }=\frac{T P}{T P+F P} \times 100 \\
& \text { Recall }=\frac{T P}{T P+F N} \times 100 \\
& F-\text { measure }=2 \times \frac{\text { Precision } \times \text { Recall }}{\text { Precision }+ \text { Recall }}
\end{aligned}
$$

Where TP represents the true positive, TN represents the true negative, FP represents the false positive, and FN represents the false negative.

Parameter settings: The aspect ratio is set as 3 in the model, the learning rate is set as $10^{-3}$, and the weight decay rate is set as 0.0005 .

System Requirement: The proposed and existing model is implemented in the system consists of an Intel i9 processor, 128 GB of RAM, 22 GB of graphics card and 3 TB hard disk. The proposed and existing models were implemented in the same environment and same data. The Python 3.7 tool was used to implement the proposed and existing model.

\section{Results}

Dysgraphia classification in childhood helps to treat the child effectively and improves selfconfidence of the child. Few existing methods involve in the dysgraphia classification based on machine learning techniques and have the limitation of overfitting problem. The existing methods have the limitations of lower efficiency in classification and non-discrimination word is not considered in the model.

This section provides a detailed description of the dataset, metrics, system requirements and results.

\subsection{Quantitative analysis}

In quantitative analysis, standard machine learning models and deep learning models were compared with the proposed NDR-R2CNN model in dysgraphia classification.

The proposed NDR-R2CNN model is evaluated for dysgraphia and compared with the existing methods, as shown in Table 1.

The proposed NDR-R2CNN model is compared with standard classifiers such as decision trees, random forests, and SVM. The four parameters such as accuracy, precision, recall and f-measure were evaluated in this analysis. The result shows that the proposed NDR-R2CNN model has higher performance compared to the existing standard classifiers. The proposed NDR-R2CNN model has the advantage of effectively analyzing the features in the data. The proposed NDR-R2CNN model also has the advantage of considering the non-discrimination of the input data.

The decision tree has the lower efficiency in feature analysis and random forest has an overfitting problem in the less few trees.

The SVM has a lower performance in handling the imbalanced data for the classification. The proposed NDR-R2CNN method has an accuracy of

Table 1. Performance comparison with standard machine learning models

\begin{tabular}{|c|c|c|c|c|}
\hline Methods & $\begin{array}{c}\text { Accurac } \\
\mathbf{y}(\boldsymbol{\%})\end{array}$ & $\begin{array}{c}\text { Precisio } \\
\mathbf{n}(\boldsymbol{\%})\end{array}$ & $\begin{array}{c}\text { Recall } \\
\mathbf{( \% )}\end{array}$ & $\begin{array}{c}\text { F- } \\
\text { measure } \\
(\%)\end{array}$ \\
\hline $\begin{array}{c}\text { Decision } \\
\text { Tree }\end{array}$ & 82.5 & 82.3 & 82.2 & 82.24 \\
\hline $\begin{array}{c}\text { Random } \\
\text { Forest }\end{array}$ & 84.1 & 84.3 & 84.2 & 84.24 \\
\hline SVM & 85.2 & 86.1 & 86 & 86.04 \\
\hline $\begin{array}{c}\text { NDR - } \\
\text { R2CNN }\end{array}$ & 98.2 & 96.4 & 100 & 98.16 \\
\hline
\end{tabular}



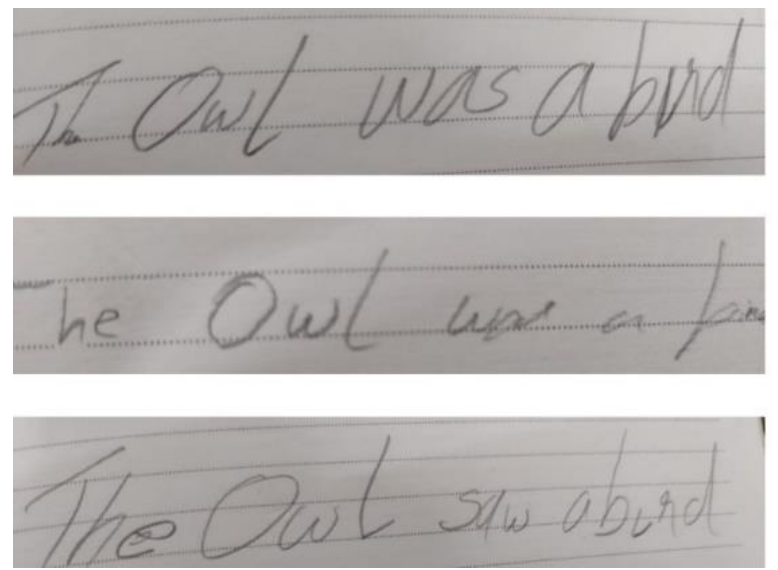

Figure. 2 The sample images of a dataset normal handwriting (top one), and dysgraphia samples (bottom two)

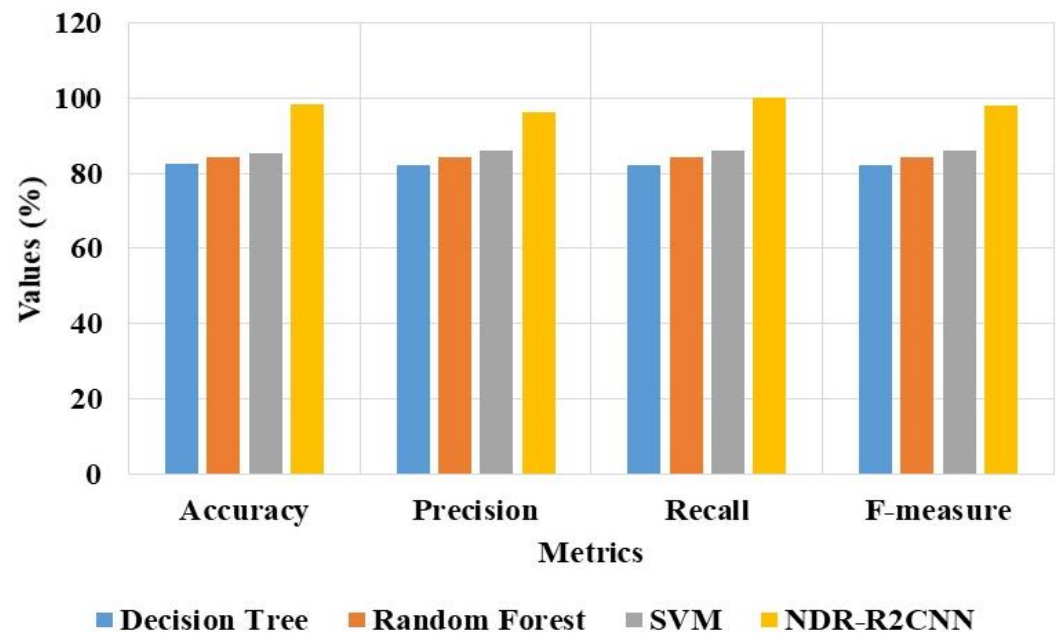

Figure. 3 Performance comparison of proposed NDR-R2CNN model and standard classifiers

$98.2 \%$ and the standard SVM classifier has $85.2 \%$ accuracy.

The proposed NDR-R2CNN model and standard classifiers were measured in terms of Accuracy, Precision, Recall and F-measure, as shown in Fig. 3. The result shows that the proposed NDR-R2CNN model has significantly higher performance compared to standard classifiers. The proposed NDRR2CNN model has the advantages of effective feature analysis and considers non-discriminative word analysis. The decision tree model has a lower efficiency in feature analysis and doesn't able to handle more data. The random forest method has the overfitting problem when the number of trees is less and lower efficiency when the number of trees is low.

The SVM model has lower efficiency in imbalance data. The proposed NDR-R2CNN model has a precision of $96.4 \%$ and SVM classifiers have $86.1 \%$ precision.

The proposed NDR-R2CNN model and deep learning models such as LSTM, RPN, fast RCNN, Flfaster RCNN, and R2CNN models were compared
Table 2. Deep learning model comparison

\begin{tabular}{|c|c|c|c|c|}
\hline $\begin{array}{c}\text { Method } \\
\text { S }\end{array}$ & $\begin{array}{c}\text { Accuracy } \\
(\%)\end{array}$ & $\begin{array}{c}\text { Precisi } \\
\text { on }(\%)\end{array}$ & $\begin{array}{c}\text { Recall } \\
(\%)\end{array}$ & $\begin{array}{c}\text { F- } \\
\text { measure } \\
(\%)\end{array}$ \\
\hline LSTM & 90.1 & 91.4 & 91.3 & 91.34 \\
\hline RPN & 88.2 & 86.5 & 86.3 & 86.39 \\
\hline $\begin{array}{c}\text { Fast } \\
\text { RCNN }\end{array}$ & 91.8 & 91.2 & 91.1 & 91.14 \\
\hline $\begin{array}{c}\text { Faster } \\
\text { RCNN }\end{array}$ & 92.1 & 91.7 & 91.5 & 91.59 \\
\hline R2CNN & 94.2 & 92.1 & 91.5 & 91.79 \\
\hline $\begin{array}{c}\text { NDR- } \\
\text { R2CNN }\end{array}$ & 98.2 & 96.4 & 100 & 98.16 \\
\hline
\end{tabular}

in Table 2. The result shows that the proposed NDRR2CNN model has a higher performance in classification. The proposed NDR-R2CNN model has the advantages of non-discrimination word analysis and effective feature analysis. The existing models have the limitations of overfitting problems that affect the efficiency of the model. The proposed NDR-R2CNN model has an accuracy of $98.2 \%$ and the existing R2CNN model has $94.2 \%$ accuracy. 


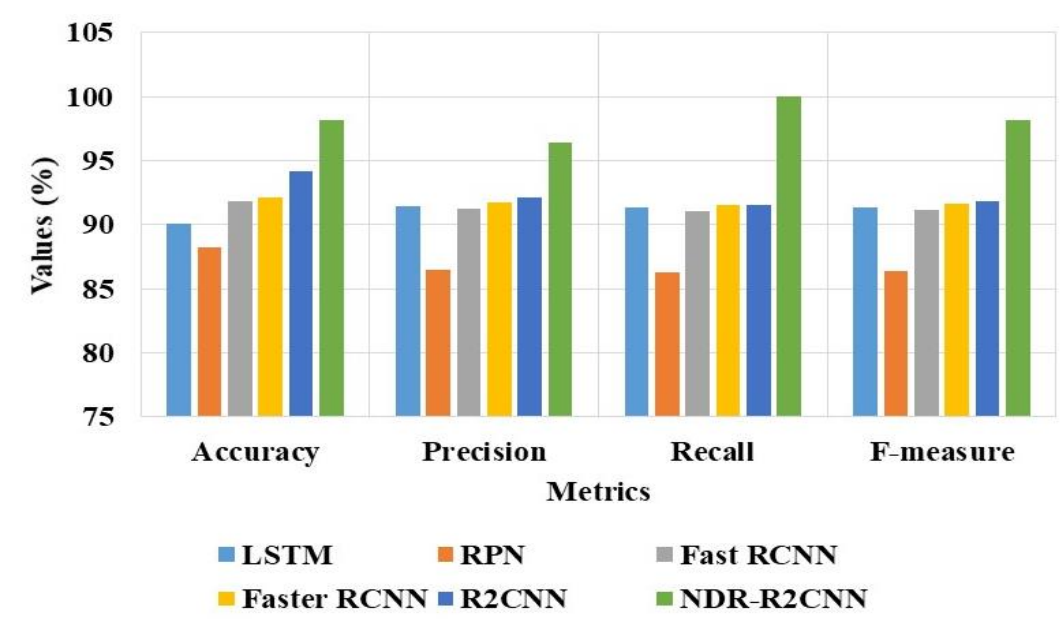

Figure. 4 Deep learning comparative analysis of dysgraphia classification

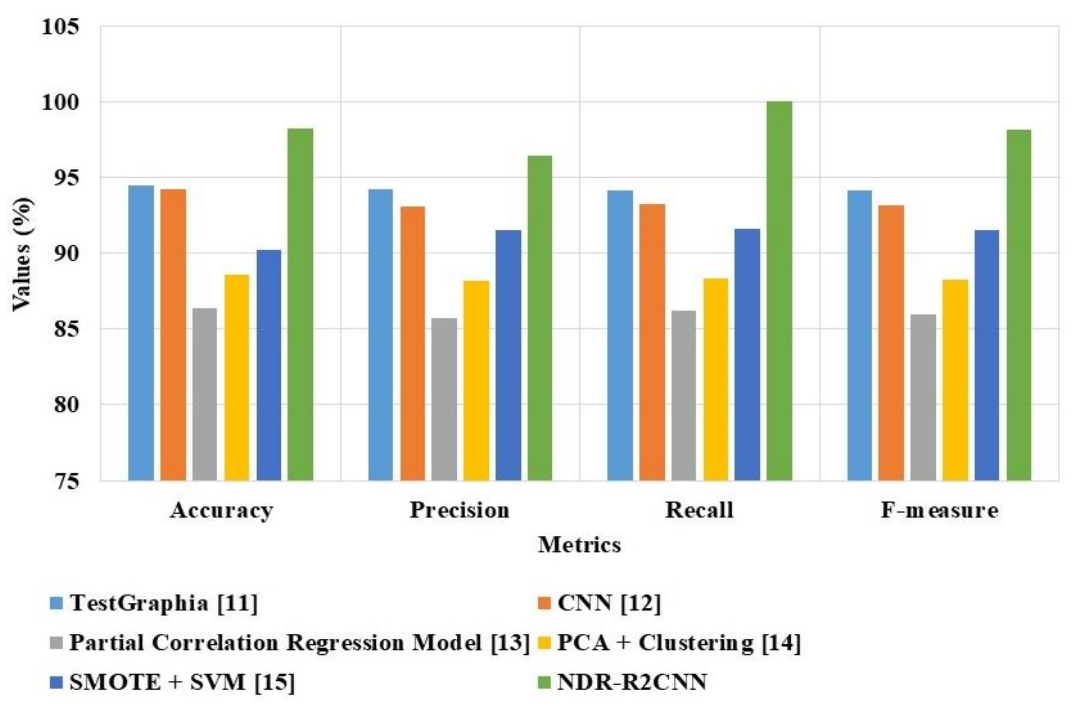

Figure. 5 Comparative analysis

The proposed NDR-R2CNN model and other deep learning models such as LSTM, RPN, fast RCNN, faster RCNN, and R2CNN were compared in Fig. 4. The result shows that the proposed NDRR2CNN model has a higher efficiency compared to other deep learning models. The proposed NDRR2CNN model has the advantages of effective feature analysis and non-discrimination feature analysis in the model. The proposed NDR-R2CNN model has a precision of $96.4 \%$ and the R2CNN model has $92.1 \%$ precision.

The proposed NDR-R2CNN model is evaluated in the dysgraphia classification and compared with existing methods [11-15]. Various existing methods were applied for the classification of dysgraphia and achieved considerable performance.

The proposed NDR-R2CNN model is compared with existing methods such as TestGraphia [11], CNN [12], Partial Correlation Regression Model [13], PCA + Clustering [14], and SMOTE + SVM [15], as shown in Table 3 . The result shows that the proposed
NDR-R2CNN has higher performance compared to existing methods. The proposed NDR-R2CNN model has the advantage of effective feature analysis and non-discrimination feature analysis.

Table 3. Comparative analysis on collected dataset

\begin{tabular}{|l|l|l|l|l|}
\hline \multicolumn{1}{|c|}{ Methods } & $\begin{array}{c}\text { Accura } \\
\text { cy } \mathbf{( \% )}\end{array}$ & $\begin{array}{c}\text { Precisi } \\
\text { on } \mathbf{( \% )}\end{array}$ & $\begin{array}{c}\text { Rec } \\
\text { all } \\
\mathbf{( \% )}\end{array}$ & $\begin{array}{c}\text { F- } \\
\text { measu } \\
\text { re } \\
(\%)\end{array}$ \\
\hline $\begin{array}{l}\text { TestGraphia } \\
{[11]}\end{array}$ & 94.5 & 94.2 & 94.1 & 94.14 \\
\hline CNN [12] & 94.2 & 93.1 & 93.2 & 93.14 \\
\hline $\begin{array}{l}\text { Partial } \\
\text { Correlation } \\
\text { Regression } \\
\text { Model [13] }\end{array}$ & 86.4 & 85.7 & 86.2 & 85.94 \\
\hline $\begin{array}{l}\text { PCA + } \\
\text { Clustering [14] }\end{array}$ & 88.6 & 88.2 & 88.3 & 88.24 \\
\hline $\begin{array}{l}\text { SMOTE + SVM } \\
\text { [15] }\end{array}$ & 90.2 & 91.5 & 91.6 & 91.54 \\
\hline NDR-R2CNN & 98.2 & 96.4 & 100 & 98.16 \\
\hline
\end{tabular}


Table 4. Comparative analysis on standard dysgraphia

\begin{tabular}{|l|l|}
\hline \multicolumn{2}{|c}{ dataset } \\
\hline Methods & $\begin{array}{l}\text { Accuracy } \\
(\%)\end{array}$ \\
\hline SVM [9] & 78.8 \\
\hline TestGraphia [11] & 80.2 \\
\hline CNN [12] & 92.4 \\
\hline Partial Correlation Regression Model [13] & 87.3 \\
\hline PCA + Clustering [14] & 88.4 \\
\hline SMOTE + SVM [15] & 93.2 \\
\hline NDR-R2CNN & 96.5 \\
\hline
\end{tabular}

The balancing parameters are applied in the loss function to balance the class in the training and eliminate the irrelevant features to solve the overfitting problem. The TestGraphia [11] model has a lower efficiency in feature analysis and CNN [12] has an overfitting problem in the training process. The SMOTE and SVM [15] model has effectively handled the imbalance data and lower efficiency in feature analysis. The proposed NDR-R2CNN model has $98.2 \%$ accuracy and the existing CNN model has $94.2 \%$ accuracy.

The proposed NDR-R2CNN model is compared with existing methods in dysgraphia classification, as shown in Fig. 5. The result shows that the proposed NDR-R2CNN model has higher efficiency in dysgraphia classification compared to existing methods. The proposed NDR-R2CNN model has the advantage of effective feature analysis and nondiscrimination feature analysis in the model. The proposed NDR-R2CNN model has $96.4 \%$ precision and the existing CNN [12] model has $93.1 \%$ precision.

\subsection{Comparative Analysis in standard Dysgraphia dataset}

The proposed method and existing methods were evaluated in the standard dysgraphia dataset and compared in Table 4.

The proposed NDR-R2CNN model is compared with existing methods in the standard Dysgraphia dataset, as shown in Table 4. The SVM, CNN, clustering methods have the limitation of imbalance data and overfitting problems in the classification. The NDR-R2CNN model has the balancing parameter to handle the imbalance data and reduces the overfitting problem. The NDR method provides the complete input data that helps to compare with the target data for better understanding. The NDRR2CNN model has $96.5 \%$ accuracy in the standard Dysgraphia dataset and CNN [12] model has $92.4 \%$ accuracy in the standard dysgraphia dataset.

\section{Conclusion}

Dysgraphia detection in childhood helps to effectively treat them and also prevent low selfesteem. Few methods were applied to machine learning and deep learning techniques for dysgraphia classification. Existing methods have the limitations of lower efficiency in feature analysis and overfitting problems. In this research, the NDR-R2CNN model is proposed to effectively detect dysgraphia and it has the advantage of effective feature analysis and nondiscrimination word analysis in the model. The balancing parameters are applied in the loss function to eliminate the irrelevant features to avoid the overfitting problem. The collected children's data were used to evaluate the proposed and existing model. The result shows that the proposed NDRR2CNN model has the advantage of $98.2 \%$ accuracy and the existing $\mathrm{CNN}$ has $94.2 \%$ accuracy. The future work of this method involves developing the attention layer to improve the accuracy of the classification.

\section{Conflicts of interest}

The authors declare no conflict of interest.

\section{Author contributions}

The paper conceptualization, methodology, software, validation, formal analysis, investigation, resources have been done by $1^{\text {st }}$ author. Data curation, writing - original draft preparation, writing - review and editing, visualization, have been done by $2^{\text {nd }}$ author. The supervision and project administration, have been done by $3^{\text {rd }}$ author.

\section{References}

[1] S. Rosenblum and G. Dror, "Identifying developmental dysgraphia characteristics utilizing handwriting classification methods", IEEE Transactions on Human-Machine Systems, Vol. 47, No. 2, pp. 293-298, 2019.

[2] P. D. Antrassi, I. Perrone, A. Cuzzocrea, and A. Accardo, "A composite methodology for supporting early-detection of handwriting dysgraphia via big data analysis techniques", In: Proc. of International Conference on Intelligent Interactive Multimedia Systems and Services, pp. 241-253, 2019.

[3] S. D. Mayes, S. S. Frye, R. P. Breaux, and S. L. Calhoun, "Diagnostic, demographic, and neurocognitive correlates of dysgraphia in students with ADHD, autism, learning disabilities, and neurotypical development", 
Journal of Developmental and Physical Disabilities, Vol. 30, No. 4, pp. 489-507, 2018.

[4] T. Asselborn, T. Gargot, L. Kidziński, W. Johal, D. Cohen, C. Jolly, and P. Dillenbourg, "Automated human-level diagnosis of dysgraphia using a consumer tablet", NPJ Digital Medicine, Vol. 1, No. 1, pp. 1-9, 2018.

[5] Y. Tao and B. Rapp, "The effects of lesion and treatment-related recovery on functional network modularity in post-stroke dysgraphia", NeuroImage: Clinical, Vol. 23, p. 101865, 2018.

[6] P. W. Samodro and S. W. Sihwi, "March. Backpropagation Implementation To Classify Dysgraphia In Children", In: Proc. of 2019 International Conference of Artificial Intelligence and Information Technology, $\mathrm{pp}$. 437-442, 2019.

[7] C. Lopez, C. Hemimou, B. Golse, and L. V. Douret, "Developmental dysgraphia is often associated with minor neurological dysfunction in children with developmental coordination disorder (DCD)", Neurophysiologie Clinique, Vol. 48, No. 4, pp. 207-217, 2018.

[8] C. Taleb, L. L. Sulem, C. Mokbel, and M. Khachab, "Detection of Parkinson's disease from handwriting using deep learning: a comparative study", Evolutionary Intelligence, pp. 1-12, 2020.

[9] P. Drotár and M. Dobeš, "Dysgraphia detection through machine learning", Scientific reports, Vol. 10, No. 1, pp. 1-11, 2020.

[10] L. Deschamps, C. Gaffet, S. Aloui, J. Boutet, V. Brault, and E. Labyt, "Methodological issues in the creation of a diagnosis tool for dysgraphia", NPJ Digital Medicine, Vol. 2, No. 1, pp. 1-3, 2019.

[11] G. Dimauro, V. Bevilacqua, L. Colizzi, and D. D. Pierro, "TestGraphia, a software system for the early diagnosis of dysgraphia", IEEE Access, Vol. 8, pp. 19564-19575, 2020.

[12] M. Gazda, M. Hireš, and P. Drotár, "MultipleFine-Tuned Convolutional Neural Networks for Parkinson's Disease Diagnosis From Offline Handwriting", IEEE Transactions on Systems, Man, and Cybernetics: Systems, 2021.

[13] J. Mucha, J. Mekyska, Z. Galaz, M. F. Zanuy, K. L. Ipina, V. Zvoncak, T. Kiska, Z. Smekal, L. Brabenec, and I. Rektorova, "Identification and monitoring of Parkinson's disease dysgraphia based on fractional-order derivatives of online handwriting", Applied Sciences, Vol. 8, No. 12, pp. 2566, 2018.

[14] T. Asselborn, M. Chapatte, and P. Dillenbourg, "Extending the spectrum of dysgraphia: A data driven strategy to estimate handwriting quality", Scientific Reports, Vol. 10, No. 1, pp. 1-11, 2020.

[15] R. Lamba, T. Gulati, K. A. A. Dhlan, and A. Jain, "A systematic approach to diagnose Parkinson's disease through kinematic features extracted from handwritten drawings", Journal of Reliable Intelligent Environments, pp. 1-10, 2021.

[16] K. Zolna, T. Asselborn, C. Jolly, L. Casteran, W. Johal, and P. Dillenbourg, "The dynamics of handwriting improves the automated diagnosis of dysgraphia", arXiv preprint arXiv: 1906.07576, 2021.

[17] X. Sun, P. Wu, and S. C. Hoi, "Face detection using deep learning: An improved faster RCNN approach", Neurocomputing, Vol. 299, pp. 42$50,2018$.

[18] S. Wan and S. Goudos, "Faster R-CNN for multi-class fruit detection using a robotic vision system", Computer Networks, Vol. 168, pp. 107036, 2018.

[19] T. Haas, C. Schubert, M. Eickhoff, and H. Pfeifer, "BubCNN: Bubble detection using Faster RCNN and shape regression network", Chemical Engineering Science, Vol. 216, pp. 115467, 2020.

[20] Y. Ren, C. Zhu, and S. Xiao, "Object detection based on fast/faster RCNN employing fully convolutional architectures", Mathematical Problems in Engineering, 2018. 